\title{
COVID-19 Outbreak and Stoma Care on a Minor Island in Italy: Physically Far, Virtually Near
}

\author{
Dario D'Antonio ${ }^{1}$ (D) - Francesco Pizza ${ }^{1} \cdot$ Francesca Paola Tropeano ${ }^{2} \cdot$ Giovanni Domenico De Palma ${ }^{2}$. \\ Alberto Marvaso $^{1} \cdot$ Gaetano Luglio $^{2}$
}

Accepted: 21 July 2020 / Published online: 12 August 2020

(C) Springer Nature Switzerland AG 2020

\begin{abstract}
The current COVID-19 pandemic has considerably stressed ordinary medical resources. In such a contest, Telemedicine (TM) was relevantly recommended by several surgical societies. A specific TM-based pathway was developed emergently to care outpatients with ileo- or colostomy living on a minor island of Southern Italy (Ischia (Naples)). We retrospectively analysed a short prospective series of 23 ileo- or colostomized patients referring to a single coloproctology out-patient clinic. Patients were followed up using TM from March 3rd to April 5th 2020, when local and national policy postponed any non-emergent and nononcologic in-patient visit to a future date. Seventeen patients were eligible for observation. Tele-consulted patients were overall satisfied, with 14 of them choosing TM as their first option for a future consultation. All medical staff gave a positive feedback about TM project. The use of TM in stoma care was easily feasible in an emergent global context. To the best of our knowledge, this is the first reported experience of remote stoma care services during COVID-19 pandemic. The next challenge is to turn emergent actions into daily outpatient practice.
\end{abstract}

Keywords Stoma care $\cdot$ Telemedicine $\cdot$ Covid- $19 \cdot$ Outpatients

The COVID-19 pandemic has considerably stressed ordinary medical resources [1]. Healthcare teams worked hard worldwide to balance risks and benefits of in-house appointments for non-urgent cases. Several surgical societies provided specific procedures and guidelines, including triaging pathways and Telemedicine (TM) during times of high disease pressure. The Society of American Gastrointestinal and Endoscopic Surgeons, the European Association for Endoscopic Surgery, the American Urological Association and the European Society of Surgical Oncology relevantly recommended TM in their documents [2].

The presence of a stoma, even in a non-emergent setting, has a great impact on patients' quality of life, so medical and

This article is part of the Topical Collection on Covid-19

Dario D'Antonio

dariusque@ fastwebnet.it

1 Division of Surgery, “A. Rizzoli” Hospital, Lacco Ameno, Naples, Italy

2 Department of Gastroenterology, Endocrinology and Endoscopic Surgery, University Hospital of Naples Federico II, Naples, Italy psychological support are needed, especially at the beginning of the stoma experience.

Ischia is a very densely populated island of Southern Italy, with more than 67,000 residents on the area. About $0.14 \%$ of permanently resident population have a stoma with a total of 109 patients: 70 have a colostomy, 27 an ileostomy and 12 a urostomy/ileal conduit. Diseases requiring operations with stoma were colorectal cancer (62 (56\%)), diverticulitis (24 $(22 \%))$, inflammatory bowel disease $(21(19 \%))$ and trauma $(2(1.8 \%))$. The mean age of patients is 71 years (range 2697); male patients are 51 (46\%). All patients with ileo- or colostomy are routinely referred to the single coloproctology outpatient clinic in charge of the General Surgical Division at “A. Rizzoli” Hospital (Lacco Ameno, Naples, Italy), the only one present on the island.

In order to maintain patient's care while limiting risks of contagion for patients and medical staff during the pandemic, a specific TM-based pathway was developed. A virtual outpatient setting was planned to support stoma carriers and their caregivers on bureaucratic issues, stoma-related complications, postoperative follow-up and evaluation of medical exams (e.g. blood tests, imaging), aiming to lower the number of emergency department (ED) admissions and in-patient 
visits. As other practices have suggested [3-5], a flow chart was drawn up matching TM with the routine modalities of outpatient visits (Fig. 1).

We retrospectively analysed a short series of prospectively collected data on ileo- or colostomized patients referring to our out-patient ostomy clinic and followed up by TM. The observation period was from March 3, 2020, to April 5, 2020 , during which the local government locked down any non-emergent and non-oncologic in-patient visits. Inclusion criteria were adults older than 18 years; presence of a supporting person in case of cognitive impairment; new patients requesting a first outpatient access; established patients requesting an outpatient visit for a new problem; established patients with a scheduled follow-up visit; and patients with stoma non-medical-related issues. Exclusion criteria were TM decline, internet/smart devices unavailability, need of physical examination or radiological investigations at a preliminary call contact. TM was defined as videoconference. Patients' willingness and attitudes for TM were preliminarily explored through phone interviews, where a specific triage questionnaire was submitted. If needed or specifically requested, in-patient visits were scheduled and, then, performed, following precautions to reduce COVID-19 spread. Recommendations included mask usage, allowing only one accompanying person per patient if necessary, temperature reading before entering both inpatient and outpatient buildings and use of personal protective equipment.

Patients were considered eligible if the primary reason for their consultation could be completely solved virtually or if inperson/on-site services such as physical examination or any other procedure were expected to be numerically reduced. Eligibility for TM was evaluated by two experienced physicians during the first contact. All included patients provided an oral informed consent.

Patients approached TM with their personal smart devices or desktop/laptop computer. Medical providers used smart devices or a desktop/laptop computer from hospital or from home. The Skype platform by Microsoft ${ }^{\circledR}$ was used for the video calling; any document drawn up by the physician was saved as PDF using the Cute Professional ${ }^{\mathrm{TM}}$ PDF software, which allows to add passwords and set security options to protect the valuable documents. An e-mail address and a phone number were available for any information or communication.

During the observation period, 23 patients were telephonically interviewed for TM eligibility. Three patients refused TM due to technical/personal limitations; 1 patient shifted on the general practitioner (GP); 2 patients chose to be re-scheduled. A total of 17 patients resulted eligible for TM.

Nine patients requested a first outpatient access: 3 patients with colostomy and 6 with ileostomy. Bureaucratic and postoperative educational issues were all managed by TM in patients with colostomy. Virtual consultation was less suitable for new patients with ileostomy: 3 patients needed more than one in-person visit, with only bureaucratic and dietary issues being fully managed with TM. The reasons for in-person visit were high-output stoma management and leakage. Two patients were admitted to ED for fluid or electrolyte imbalance and bleeding. After discharge, patients were remotely followed up, resulting, anyway, in a lower number of in-person accesses.

Five established patients requested an outpatient visit for a new problem: 3 patients with colostomy and 2 with ileostomy. All patients were suited to TM. The reasons for consultation
Fig. 1 Flow chart for stoma outpatients' access during COVID-19 outbreak-TM, Telemedicine; ED, emergency department

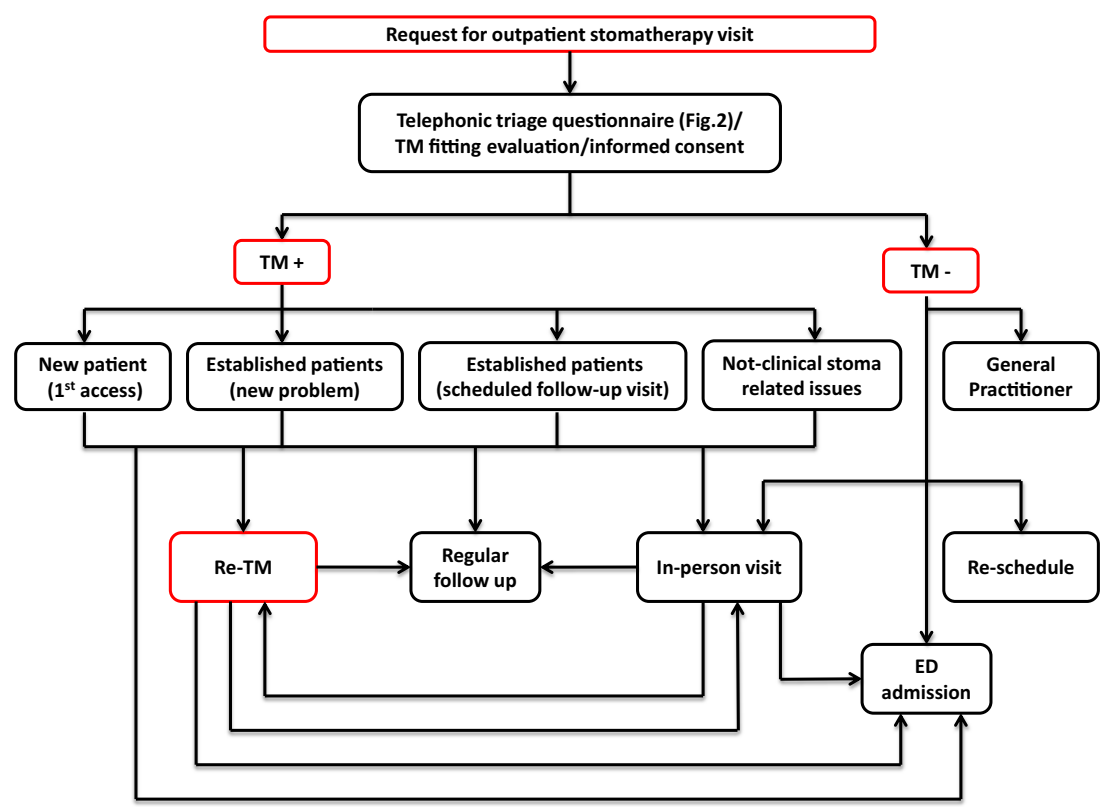


were peristomal skin modifications (ulcerations, granulomas), stoma prolapse, parastomal hernia and/or anal discharge. No patient needed an in-person access.

Among 5 established patients with a scheduled follow-up visit, 3 were fully managed through TM: 2 patients with colostomy and 1 with ileostomy. No difference in the management was encountered between ileo- and colostomy. Two patients chose to be re-scheduled.

Tele-consulted patients were globally satisfied, with 14 of them choosing TM as their first option for a future consultation. The whole medical staff gave a positive feedback about TM project.

Amid the COVID-19 pandemic, healthcare access facilities in a safe setting became a priority as social distancing was promoted worldwide. TM was tested in disasters and public health emergencies [6] and is widely used in both the postoperative period and outpatient follow-up. Haemodialysis, dermatology, orthopaedics and neurology were fields in which TM was evidence-based used and, so, implemented in some countries [7-9]. Furthermore, TM appeared a safe and feasible postoperative option for clinical and wound evaluation of patients undergoing inguinal hernia repair, cholecystectomy or appendectomy [10]. Notably, wound care and wound healing, as well as stoma care and stoma healing, are good candidates for TM because a relevant portion of the consultation is dedicated to inspection. White et al. applied TM to stoma management during the early postoperative period, concluding that patients felt comfortable with the format and overall satisfied [11]. The findings of a randomized controlled trial on stoma patients who discharged from hospital indicated that follow-up care at home via a mobile app could effectively improve the psychosocial adjustment level, self-efficacy scale and other related outcomes of stoma patients [12]. Furthermore, another randomized controlled trial (STOMPA trial) showed that both readmission rate and burden of travel were decreased by a TM project [13]. Our experience was similarly positive, reaching $82 \%$ of patients successfully followed up remotely and willing to be re-scheduled for teleconsultation in the future. A TM service is well suited for an insular contest like ours, where almost all patients refer to the same outpatient clinic to be taken in charge and followed up. Patients are often operated in 2nd-/3rd-level surgical centres on the mainland, even many miles distant from the island, so, beyond the outbreak, such a project could act as a starting point for a desirable network between local health services and 2nd-/ 3rd-level surgical centres. Besides that, some patients live in rural and uncomfortable areas of the island, where even the access to the insular outpatient clinic can be logistically troubling and takes considerable time. As elsewhere mentioned, number of transfers and its relative stress for both the patient and the caregiver could be significantly reduced [13]. In our series, among new stomized outpatients, ileostomy carriers required more frequently an in-person access, thus resulting in a poorer outcome of the TM pathway. Patient with ileostomy are, anyway, more fragile, and physical care is often essential to best manage skin problems, psychological concerns, fluid balance and stoma output. ED admission was required for 3 new ileostomized patients. Interestingly, among 5 established patients with a scheduled follow-up visit, 3 chose to be re-scheduled rather than to be followed up virtually, probably because they felt their follow-up not strictly necessary. Teleconsultation was not only related to the stoma site and stoma-related problems, but any other wound was virtually explored, if needed. In this context, during their first TM meeting, 2 new patients undergoing a recent operation complained with primary wound rather than stoma-related concerns: they were all colostomy carriers, and all their complains were remotely managed. It must, anyway, be highlighted that all recently operated patients had a postoperative in-person global check by their GP, including wound management.

Some considerations are, anyway, necessary. First, in cases of patients with no home/cell phone access to internet, we furnished medical consultations through telephone calls, and the patients did appreciate our efforts. Anyway, a previous paper on telephone follow-up of patients returning home with colostomy had showed that all examined patients benefited from the nurse-led modality of follow-up [14]. Another issue that should be further investigated is the current reimbursement rates for video visits, especially in countries like Italy, where TM represents the exception rather than the rule. This latter issue, anyway, should be addressed by central and local health governments, synergically with health institutions and hospitals.

To the best of our knowledge, this is the first reported experience of TM in stoma care during COVID-19 pandemic. The project is, anyway, still ongoing, with the challenge to turn emergent actions into daily outpatient practice.

Author's Contribution Conceptualization, Dario D'Antonio; methodology, Dario D'Antonio; formal analysis and investigation: Dario D'Antonio and Francesco Pizza; writing — original draft preparation, Dario D'Antonio and Francesco Pizza; writing - review and editing, Gaetano Luglio and Francesca Paola Tropeano; supervision, Giovanni Domenico De Palma and Alberto Marvaso; study coordination, Gaetano Luglio.

\section{Compliance with Ethical Standards}

Conflict of Interest The authors declare that they have no conflict of interest.

Ethical Approval All procedures performed in studies involving human participants were in accordance with the ethical standards of the institutional and/or national research committee and with the 1964 Helsinki declaration and its later amendments or comparable ethical standards. The referring Ethic committee is "Campania Centro". 
Informed Consent Informed consent was obtained from all individual participants included in the study.

\section{References}

1. Zou L, Ruan F, Huang M, Liang L, Huang H, Hong Z, et al. SARSCoV-2 viral load in upper respiratory specimens of infected patients. N Engl J Med. 2020;382(12):1177-9.

2. Søreide K, Hallet J, Matthews JB, Schnitzbauer AA, Line PD, Lai PBS, et al. Immediate and long-term impact of the COVID-19 pandemic on delivery of surgical services. Br J Surg. 2020. https://doi.org/10.1002/bjs.11670.

3. Price KN, Thiede R, Shi VY, Curiel-Lewandrowski C. Strategic dermatology clinical operations during the coronavirus disease 2019 (COVID-19) pandemic. J Am Acad Dermatol. 2020;82(6): e207-9. https://doi.org/10.1016/j.jaad.2020.03.089.

4. Hollander JE, Carr BG. Virtually perfect? Telemedicine for Covid19. N Engl J Med. 2020. https://doi.org/10.1056/NEJMp2003539.

5. Smith AC, Thomas E, Snoswell CL, Haydon H, Mehrotra A, Clemensen J, et al. Telehealth for global emergencies: implications for coronavirus disease 2019 (COVID-19). J Telemed Telecare. 2020. https://doi.org/10.1177/1357633X20916567.

6. Lurie N, Carr BG. The role of telehealth in the medical response to disasters. JAMA Intern Med. 2018;178:745-6.

7. Buvik A, Bugge E, Knutsen G, Småbrekke A, Wilsgaard T. Patient satisfaction with remote orthopaedic consultation by using telemedicine: a randomised controlled trial. J Telemed Telecare. 2018;21: $1-9$.
8. Müller KI, Alstadhaug KB, Bekkelund SI. A randomized trial of telemedicine efficacy and safety for nonacute headaches. Neurology. 2017;89:153-62.

9. Norum J, Pedersen S, Størmer J, Rumpsfeld M, Stormo A, Jamissen N, et al. Prioritisation of telemedicine services for large scale implementation in Norway. J Telemed Telecare. 2007;13: 185-92.

10. Nikolian VC, Williams AM, Jacobs BN, Kemp MT, Wilson JK, Mulholland MW, et al. Pilot study to evaluate the safety, feasibility, and financial implications of a postoperative telemedicine program. Ann Surg. 2018;268:700-7.

11. White T, Watts P, Morris M, Moss. Virtual postoperative visits for new ostomates. J. Comput Inform Nurs. 2019;37(2):73-9.

12. Wang QQ, Zhao J, Huo XR, Wu L, Yang LF, Li JY, et al. Effects of a home care mobile app on the outcomes of discharged patients with a stoma: a randomised controlled trial. J Clin Nurs. 2018;27(19-20):3592-602. https://doi.org/10.1111/jocn.14515.

13. Augestad KM, Sneve AM, Lindsetmo RO. Telemedicine in postoperative follow-up of STOMa PAtients: a randomized clinical trial (the STOMPA trial). Br J Surg. 2020;107:509-18.

14. Zheng MC, Zhang JE, Qin HY, Fang YJ, Wu XJ. Telephone follow-up for patients returning home with colostomies: views and experiences of patients and enterostomal nurses. Eur J Oncol Nurs. 2013;17(2):184-9. https://doi.org/10.1016/j.ejon.2012.05. 006.

Publisher's Note Springer Nature remains neutral with regard to jurisdictional claims in published maps and institutional affiliations. 